\title{
The mental well-being and coping strategies of Canadian adolescents during the COVID-19 pandemic: a qualitative, cross-sectional study
}

\author{
Kendra Nelson Ferguson PhD, Stephanie E. Coen PhD, Danielle Tobin PhD, Gina Martin PhD, \\ Jamie A. Seabrook PhD, Jason A. Gilliland PhD
}

Abstract

Background: Qualitative research is lacking on the mental well-being of adolescents during the COVID-19 pandemic. The aim of this study was to explore the feelings and emotions adolescents experienced during the first wave of the COVID-19 pandemic and the coping strategies they identified and employed to manage those emotions.

Methods: Participants living in Canada aged 13-19 years were recruited through social media platforms and youth-serving organizations. Qualitative data were gathered from 2 open-ended questions included in a youth-informed cross-sectional online survey: "What feelings and emotions have you experienced around the pandemic?" and "What coping strategies have you used during the pandemic?" We collected data from June 2020 to September 2020. A summative content analysis was undertaken to analyze survey responses inductively.

Results: A total of 1164 open-ended responses from Canadian adolescents ( $n=851$; mean age 15.6, standard deviation 1.7, yr) were analyzed. We identified 3 major themes within the category of feelings and emotions associated with the pandemic: sociospatial and temporal disconnections, emotional toll of the pandemic and positives amid the pandemic. Within the category of coping strategies used during the pandemic, 2 major themes were identified: connecting online and outdoors, and leisure and health-promoting activities.

Interpretation: Although the emotional toll of the first wave of the COVID-19 pandemic is evident, participants in our study adopted various positive coping strategies to mitigate their distress, including physical activity, safe peer interactions and hobbies. The results have important implications for public health policy and practice during pandemic times, emphasizing the importance of accessible mental health resources for those experiencing psychological distress.

n Mar. 12, 2020, in response to the global COVID-19 pandemic, Canada implemented various public health interventions, including physical distancing measures, stay-at-home orders and quarantines to reduce spreading of the virus. Although some elements of the interventions varied by province, most Canadians were in some form of "lockdown" between March and September 2020. ${ }^{1}$ The substantial societal changes and disruption to adolescents' daily routines likely make them vulnerable to impacts on their well-being and development. ${ }^{2-4}$ School closures, home isolation, cancelled social gatherings and events, loss of important milestones (e.g., graduation and school social events), uncertainty about the future, and substantial disruptions to peer interactions and experiences may critically affect the emotional, behavioural and identity development of adolescents. ${ }^{4-8}$ Thus, the complex stressors endured could be associated with adverse mental health outcomes. ${ }^{4}$

There has been an increase in anxiety and depression among children and adolescents from continuous social isolation from COVID-19.9 Research investigating pandemic effects on youth aged 14-28 years in Ontario, Canada, found an increase in mental health concerns (i.e., depression, anxiety and self-harm) during the early stages of COVID-19. ${ }^{10}$ A survey among youth in India reported moderate-to-severe stress, anxiety and depression symptoms during the pandemic. ${ }^{11}$ Research examining Nigerian parents' perceptions of the psychosocial effect of pandemic restrictions on children showed that those with limited Internet access expressed unhappiness, stress and anxiety. ${ }^{12}$ Stay-at-home orders in China resulted in higher rates of anxiety and depressive symptoms among children, ${ }^{13}$ a decrease in social support from peers and a higher prevalence of mental health problems. ${ }^{14}$

Competing interests: Kendra Nelson Ferguson and Gina Martin received postdoctoral trainee support from the Children's Health Foundation through the Children's Health Research Institute. No other competing interests were declared.

This article has been peer reviewed.

Correspondence to: Jason Gilliland, jgillila@uwo.ca

CMAJ Open 2021 November 16. DOI:10.9778/cmajo.20210042 
Pandemic conditions have created new challenges for young people's coping capacities as well as their development of coping skills. These are developed through different processes and environmental contexts, ${ }^{15}$ and often benefit from positive relationships, cohesive families and challenging contexts. ${ }^{16}$

Qualitative research is lacking on the mental well-being of adolescents during the COVID-19 pandemic. We sought to qualitatively examine mental well-being outcomes and coping strategies among adolescents related to the pandemic. By examining adolescents' feelings and emotions through openended questions, which allow respondents to express themselves in their own words, we gain a deeper understanding of their experiences during this challenging time. We sought to explore the feelings and emotions adolescents experienced around the COVID-19 pandemic and the coping strategies they identified and employed to manage those emotions.

\section{Methods}

\section{Study design and setting}

A youth-informed cross-sectional online survey (e-survey) called QuaranTEENing was distributed to gain an understanding of the impacts of COVID-19 restrictions (e.g., physical distancing and stay-at-home orders) on adolescents' mental and emotional well-being. This study was reported according to the Checklist for Reporting Results of Internet E-Surveys. ${ }^{17}$ In this paper, we report on the findings of 2 optional, open-ended questions included in the survey.

\section{Participants}

Participants were recruited through survey advertisements that were created and posted on various social media networks (e.g., Instagram and Twitter; Appendix 1, available at www. cmajopen.ca/content/9/4/E1013/suppl/DC1). In addition, recruitment emails containing flyer advertisements were sent to several youth-serving organizations in Canada (e.g., YMCA; Appendix 1). Recruitment material contained a weblink directing potential participants to the e-survey in Qualtrics software, which began with a letter of information outlining study details and eligibility (i.e., aged 13-19 yr and living in Canada). Exclusion criteria included ineligible age, living outside Canada, and inability to complete the survey in English or French. Consent was implied if adolescents completed the survey.

We collected data from June to September 2020 (in Canada, in-person schooling stopped March 2020). On completion, participants were entered into a draw for a $\$ 250$ gift card. Because of the nature of this study, participants were provided with a list of mental health resources (e.g., Wellness Together Canada: Mental Health and Substance Use Support) to access for support, at the start and end of the survey.

\section{Survey}

The survey was developed by a multidisciplinary team (K.N.F, S.E.C, D.T, G.M, J.A.S. and J.A.G., with specialties in health geography, health science, epidemiology and biostatistics) based on discussions and early literature on the
COVID-19 pandemic and youth health and well-being. ${ }^{10,18}$ Members of the Human Environments Analysis Laboratory Youth Advisory Council $(n=18),{ }^{19}$ which integrates youth voices, perspectives and expertise into our research, piloted the survey for readability and usability. After feedback was received, minor adjustments were made to enhance the survey's functionality before fielding.

This paper focuses on findings from 2 optional, openended questions: "What feelings and emotions have you experienced around the pandemic?" and "What coping strategies have you used during the pandemic?" These questions were placed after Likert-scale questions asking about selfrated mental health, stress and psychological distress, before and during COVID-19 restriction measures.

\section{Data analysis}

We conducted a summative content analysis to analyze survey responses inductively. This approach is commonly used for open-ended surveys with a large number of responses ${ }^{20,21}$ and involves comparing content and counting keywords, followed by interpretation of the context. Two researchers (K.N.F. and D.T.) worked independently to group similar responses into themes, where subthemes were constructed from repeating ideas. ${ }^{22}$ The researchers removed any responses deemed irrelevant (e.g., "N/A" or "none"). After the 2 researchers came to a consensus on the themes and subthemes, a third researcher (S.E.C.) provided a check on the plausibility of themes and subthemes, and the consistency of analysis, adding to the rigorous approach. ${ }^{23}$ Any discrepancies were discussed as a group, where themes and subthemes were refined until agreement was reached. Keywords were then extracted and quantified using frequency counts ${ }^{20}$ verified using NVivo 12 Pro. Quantifying responses allows for inferences to be made about the magnitude of an issue considered important to participants. ${ }^{23}$

\section{Ethics approval}

Study approval was obtained from Western University's nonmedical research ethics board (\#115958).

\section{Results}

A total of 1228 open-ended responses (489 responses to the first question on feelings and emotions and 739 responses to the second that addressed coping strategies) were received. After irrelevant responses were removed, 449 responses for the first question and 715 for the second were analyzed. Participants included 851 Canadian adolescents $(71.1 \%$ female, $25.9 \%$ male, $1.6 \%$ trans or nonbinary, and $1.4 \%$ unknown or other [e.g., demiboy, genderfluid, agender]); the age range was 13-19, and the mean age was 15.6 (standard deviation [SD] 1.7) years (Table 1). Most respondents $(61.5 \%)$ lived in large urban centres (population $\geq 100000$ ), $13.6 \%$ in rural areas (population <1000), $10.9 \%$ in medium-sized population centres (population 30000-99999) and 9.9\% in small population centres (population 1000-29999). The e-survey took about 20 minutes to complete. 


\begin{tabular}{|lc|}
\hline $\begin{array}{l}\text { Table 1: Demographic characteristics of survey respondents } \\
(\boldsymbol{n}=\mathbf{8 5 1})\end{array}$ \\
\hline $\begin{array}{l}\text { No. }(\%) \text { of } \\
\text { Characteristic }\end{array}$ \\
\hline Gender \\
\hline Female \\
\hline Male \\
\hline Trans or nonbinary
\end{tabular}

Within the 2 broader categories under analysis (feelings and emotions associated with the pandemic; coping strategies used during the pandemic), 5 major themes and 22 subthemes were identified. In this paper, we include quotes from 86 participants (65 female, 20 male and 1 trans or nonbinary); the age range of these participants was 13-19 (mean 15.8, SD 1.6) years.

\section{Feelings and emotions associated with the pandemic}

Analysis of this category generated 3 themes: sociospatial and temporal disconnections, emotional toll of the pandemic, and positives amid the pandemic. The number of participants supporting each theme can be found in their respective boxes below.

\section{Sociospatial and temporal disconnections}

This theme reflects 5 subthemes that demonstrate a range of disconnections adolescents experienced across time, place and social relationships (Box 1). Participants called attention to the challenges navigating stay-at-home measures, namely, online school while isolated at home. Learning online was perceived to be more difficult owing to isolation, missing friends, decreased motivation, increased workload and tensions at home.

Missing in-person interaction, support and connection contributed to the despair participants experienced. Participants were craving in-person interaction, realizing the need to see their friends regularly for mental and emotional health. Feeling like the support they used to rely on was pulled out from underneath them and how they would love to hug friends and family emphasized the importance of support and connection for this demographic.

As momentous life events were derailed, participants feared how missing milestones and opportunities (e.g., high school graduation, beginning postsecondary education, and sport) would affect them now and in the future. Further, an overwhelming sense of disconnection, loneliness and isolation was common for many participants who noted feeling extremely alone, shut off and disconnected from the world.

Many participants felt uneasy and distressed about the future because of unknown consequences of the pandemic and uncertainty of how life was going to end up, causing many adolescents to feel anxious and nervous. Participants linked the various disconnections to their mental and emotional well-being, placing emphasis on the urgency for peer interaction, connection, support and experience.

\section{Emotional toll of the pandemic}

This theme shows the damaging emotional toll of the pandemic in participants' lives (Box 2). Adolescents articulated their concern for safety, including being uncomfortable with physical touch, fear of public spaces, worry for others' wellbeing and feeling anxious to leave the house. Feeling broken down and at a loss was the most common sentiment among participants $(n=126)$, who reported a loss of independence, happiness and motivation. Feeling trapped and not excited to wake up, with thoughts of worthlessness and hopelessness, was also evident.

Participants experienced a cycle of emotions, where emotions switched faster than usual and they could be having fun one second, then angry the next. Finally, although mentioned with a low frequency $(n=4)$, some participants disclosed a struggle with suicidal thoughts, attributing these thoughts to isolation and loneliness. Through these subthemes (cycle of emotions and suicidal thoughts), it is evident that participants were extremely vulnerable to the harmful effects of the pandemic, calling attention to the mental and emotional distress endured.

\section{Positives amid the pandemic}

Despite the overwhelming negative mental impacts, participants did find positives amid the pandemic (Box 3). This theme reflects 3 subthemes. Participants $(n=82)$ expressed feeling more at ease, with less stress and pressure from daily demands, and that it was a relief to be able to relax from schoolwork- and extracurricular-related stress. A small number 


\section{Box 1: Sociospatial and temporal disconnections $(n=295)$}

Challenges navigating stay at home orders $(n=19)$

- The way school is sucks. It's more stressful and I feel I could give up on work easily. (P407)

- I miss my friends and feel sad not to have a social life. It's been hard at home to stay and do learning online, and a bit isolating. (P823)

- Physical distancing is hard because I am home again with my parents. It's been tough being back here. It wasn't the perfect household. (P875)

- Being stuck with the same people is really hard and there tends to be a lot of tension. (P321)

Missing in-person interactions, support and connections $(n=68)$

- I wish I could just hug my friends and hang out with them like no problem. I feel as though l've taken physical touch for granted. (P39)

- I never realized how much I needed to see my friends regularly for my mental and emotional health and l've missed physical closeness more then l'd originally imagined I would. (P1879)

- It was very hard since my usual coping mechanisms weren't there; i.e., teachers, friends, other adults, etc. I don't often share my feelings with my parents so this was hard. (P1700)

- It has been near to impossible to be away from my friends who are my support system. (P211)

Missing milestones and opportunities $(n=33)$

- $\quad$ As a 2020 graduate, I was sad that I won't be able to experience important milestones such as graduation, prom, and the last day of school with my friends. (P454)

- I am very upset about losing grad[e] 12 grad/prom, feeling a sense of loss of everything I don't get to experience. (P58)

- I feel sad as I miss my friends and teachers. I also feel sad because of missed opportunities, and plans I had been looking forward to. I feel sad when I read the news, and think about being unable to say goodbye to some people from school who are not returning next year. I am worried about how my grade 12 year and university will go, and that I will have to miss more opportunities. (P672)

- Physical distancing has definitely ruined our year. I feel I missed out on making so many memories with my family and friends and I fear that I will never get this time back. (P840)

Disconnected, isolated and lonely $(n=95)$

- $\quad$ I've been feeling disconnected and isolated from people my age and without friends. (P1804)

- I feel less connected to others and lonelier, online communication isn't as easy or enjoyable as in person. (P198)

- I feel very isolated and I feel as if there is no light at the end of the tunnel. Sometimes it just feels as if I'm going through the motion of life but not actually living it. (P1915)

- Physical distancing also made me feel more isolated and disconnected from the rest of the world. Felt like I was alone. (P1908)

Uneasy and distressed about the future $(n=80)$

- The fact that everything is unclear about the future, even more than normally, gives me quite a lot of anxiety and since some exams from uni are replanned I felt quite stressed too and if something unexpected happens, it can really throw me off in some kind of panic-cry. (P433)

- I I am scared of everything going back to normal and me being left behind, unprepared for yet another change. (P1694)

- $\quad$ Thinking about the future also makes me feel very anxious and nervous, where before I was excited. When I think of my future, I feel more of a sense of dread and a lot of worry and a lot of hopelessness, as I'm realizing that the experiences I had imagined for myself are now not going to happen due to corona. (P817)

- Everything feels uncertain, but I am at a point in my life where I have to make many decisions very quickly about things that will affect my future for decades to come. I am not equipped to do so. (P2027) of participants mentioned that more time at home and access to online platforms enabled them to feel more connected to friends and loved ones. Further, reduced demands allowed for participants' selfreflection, growth and care, particularly time to focus on themselves and organize their thoughts, adding to perceptions of improved mental well-being. In the face of adversity, many adolescents said that the pandemic allowed for more connection with friends and family, and time to focus on themselves.

\section{Coping strategies used during the pandemic}

In addition to identifying emotions and feelings, participants noted adopting various coping strategies to help them through these uncertain times, resulting in 2 themes: connecting online and outdoors (3 subthemes), and leisure and health-promoting activities (7 subthemes).

\section{Connecting online and outdoors}

Given restrictions on physical interaction, participants noted shifting social interactions online or outdoors (Box 4). The most frequently reported $(n=228)$ way of connecting with others was through online platforms, including video calls, texting, playing video games together, and social media. Participants mentioned connecting online as a way to stay connected and help relieve the stress of the situation. With stay-at-home orders, participants mentioned that they were spending more time connecting with their families than before as a way to cope with physical distancing. This was done by engaging in more activities together, such as playing games, going for walks or just hanging out.

A small number of participants mentioned using visits with friends or family outdoors as another way to connect and socialize, while following physical distancing guidelines. Although means of connecting with others changed, the importance of connecting with others to cope with the pandemic was evident.

\section{Leisure and health-promoting activities}

This theme demonstrates the various leisure and health-promoting activities that participants engaged in to cope with the pandemic (Box 5). Physical activity was frequently reported $(n=186)$ as an important 


\section{Box 2: Emotional toll of the pandemic $(n=184)$}

Knowledge and concern for safety $(n=14)$

- I honestly don't know when l'll be able to hug one of my friends without feeling uncomfortable or that I have to take a shower immediately. (P332)

- I get really awkward, stressed and scared when people get too close to me when I'm out in public. I always have to remind my parents to not get too close to neighbours and such when they're talking, and l've become a complete germophobe when touching anything, even the groceries in my own fridge. All these kind of feelings are definitely due to COVID-19 and are not something I can see going away easily even after the pandemic is over. (P1717)

- I do feel extremely anxious if I leave the house to a public area even if I maintain my distance and wear masks and am obsessive over potential symptoms. (P2015)

- I feel afraid of folks who do not safely distance, as it feels like a breach of my (as well as their) personal safety. (P22)

Broken down and at a loss $(n=126)$

- Physical distancing, even in stage 3 has meant that l've lost a lot of independence. (P1917)

- It makes me sad and hopeless because my friends were what made me happy, and now I can't even see them. (P294)

- I feel trapped and don't feel very excited to wake up every morning. I'm tired of being at home. I feel drained all the time. (P235)

- $\quad$ Have nothing to look forward to when I wake up. (P2127)

Cycle of emotions $(n=40)$

- I remain in this cycle, break down more than I did before and I just didn't think that I would feel this way when this all started. (P614)

- I find that now in quarantine my emotions switch faster. I could be having fun one second then angry the next. (P220)

- I've noticed the negative feelings I have felt are not constant. They will all show up at one point, overwhelming me. Then life continues, and I move on for a little bit of time. Then the cycle continues. (P314)

- Some days I feel fine, other days I feel very sad, almost sick inside and I don't know why. (P752)

Suicidal thoughts $(n=4)$

- $\quad$ Felt somewhat suicidal because I couldn't see my friends and wasn't getting along with my mom and everything was building up. (P625)

- $\quad$ Suicidal every now and then. (P430)

- I have experienced suicidal thoughts. (P36)

coping mechanism and a way to feel better. This included incorporating exercise into daily routines, going for walks with family members or working on sport-specific skills. A few adolescents mentioned the importance of being outside as often as possible for fresh air. Participants also noted how finding activities and new hobbies to keep them busy and distracted from current events was critical, including video gaming, cooking and baking, arts (i.e., crafts, music and dance), reading, and watching television or movies. The leisure activities noted in this theme provided a positive way for participants to cope with the events happening around them.
Box 3: Positives amid the pandemic $(n=113)$

Feeling more at ease with less stress and pressure $(n=82)$

- $\quad$ After schools closed I could tell my mental health and even physical health started vastly improving. I have time to do things I love, I get a good amount of sleep each night, I eat properly now and I'm not stressed. (P619)

- $\quad$ Feeling significantly less stressed and pressured as before quarantine my schedule was jam packed and I rarely had a free minute. (P887)

- I am generally feeling a lot less stressed since school has been closed. School work was the biggest cause of stress in my life, and with the workload reduced and our marks not able to go down, I now have time to pace myself, learn, and I now have time to relax. (P257)

- I feel amazing and so at ease. School is so hard to maintain physically and mentally. It is incredibly draining trying to keep up with people [and] look your best and seem happy all the time. I am an introvert at heart and this quarantine has made my mental health much better and has honestly turned my life for the better. I am so happy that I don't have to put up with faking being cheery all the time at school and I can take the time to do things I love like read which I haven't have time for awhile because all my school work. (P933)

More connected to friends and loved ones $(n=11)$

- I was not overly social before COVID but since then l'd say I've gotten closer with some friends. (P119)

- $\quad$ I'm getting much closer to my family. (P219)

- I feel like I have been able to reconnect with family members. (P277)

- I feel a lot more connected to my friends. (P1909)

Self-reflection, growth and care $(n=20)$

- With less distractions, and more free time I was finally able to focus on my relationships and my mental health. Not having to go to school also gave me the chance to realize how some friends' impact was not the best for me. (P1380)

- The gap in time that this has caused has allowed me to organize how I feel and what I think about my point of view in my life for the better. (P1889)

- $\quad$ Being in quarantine has given me much more time with my thoughts and emotions. I am beginning to understand myself and try to actually figure out how to open up. (P1777)

- After physical distancing I allowed myself to take some time and focus on myself. That helped me grow out of my depressive state and frequent panic attacks. (P2013)

\section{Interpretation}

To understand adolescents' experiences during the first wave of the COVID-19 pandemic, this study explored the feelings and emotions adolescents experienced and the coping strategies employed to manage those emotions. The fact that so many adolescents were willing to share their lived experiences through 2 optional, open-ended e-survey questions shows the magnitude of the impact of the pandemic on adolescents' well-being. Our results highlight the overwhelming emotional toll of the pandemic (e.g., loneliness, disconnection and distress) and the disruption it caused to adolescents' lives. However, participants disclosed that although they faced many 


\section{Box 4: Connecting online and outdoors $(n=321)$}

Online $(n=228)$

- [I] have also been video calling family members to stay connected and help relieve the stress of the situation. Communicating and trying to stay focused helps relieve stress and anxiety. (P539)

- I FaceTime my friends a lot more than I did before. (P790)

- I've been trying to keep in contact with my friends through platforms such as FaceTime and Snapchat. (P956)

- I have been connecting with my friends and family outside my home virtually. (P66)

Connecting with family $(n=70)$

- $\quad$ l've been hanging out with my family more often. We've been having fires in our backyard and trying to stay [positive]. (P2069)

- $\quad$ My family has helped me cope with physical distancing because I'm very close with them and since now they are all home, I get to spend much more time with them. (P1848)

- $\quad$ Spending more time with my family. Playing more games as a family (e.g., board games) that we wouldn't have time for without COVID-19. (P1665)

- $\quad$ Spending time with my family are the times I most cherish during this time. (P1041)

Visits with friends and/or family outdoors $(n=23)$

- Sometimes close friends will come by my house and we will sit physical distanced and talk for a while. (P276)

- My neighbours also come to my backyard where we often have campfires at a safe distance. (P112)

- Hanging out with my friends in the outdoors. (P1977)

- $\quad$... spend some time in the backyard with my neighbours when the weather is nice. (P130)

challenges, there were positives amid the pandemic, such as feeling more at ease, with less stress and pressure, and feeling more connected with friends and loved ones. Further, results point to the positive coping strategies adolescents adopted to mitigate their distress, including health-promoting activities (e.g., physical activity), safe peer interactions (e.g., connecting online or outside) and hobbies (e.g., cooking and baking).

The negative feelings and emotions disclosed reflect subjective responses common during exposure to a traumatic event, ${ }^{4,24}$ with the most prevalent sentiments around disconnection, isolation, loneliness, feeling broken down and at a loss, and experiencing distress about the future. When adolescents are exposed to trauma from multiple stressors endured (e.g., substantial life disruption, threat to personal safety and health, and loss of support systems and experiences), they become more vulnerable to adverse mental health outcomes. ${ }^{4,14,25}$ Thus, consistent with previous research, ${ }^{2-4,7,8}$ results indicate that the negative emotions experienced by participants can be linked to a decline in mental well-being.

The disconnections adolescents experienced during this time point to the need for interventions that leverage adolescent friend and peer networks during pandemic times. In this study during the first COVID-19 wave in Canada, and relevant to many segments of Canada, adolescents were e-learning

\section{Box 5: Leisure and health promoting activities $(n=449)$}

Physical activity $(n=186)$

- I have been working out and doing a lot of yoga. (P1939)

- $\quad$... doing lots of physical activities and feeling better. (P1944)

- I've been walking my dogs and dancing. Physical activity has been really helpful. (P795)

- $\quad$... practising my shot, working on baseball skills with my dad and my brothers. (P120)

Being outside $(n=24)$

- I've been going to the beach, which helps me feel relaxed and at ease. (P2029)

- $\quad$ Keeping myself busy by going outside and getting fresh air instead of staying inside all day. (P760)

- $\quad$... spending a lot of time outside in the forest or beach. (P881)

- $\quad$... going in my backyard for fresh air. (P779)

Video games $(n=76)$

- $\quad$ I have been playing my PlayStation 4 with my friends so I don't get lonely. (P1909)

- $\quad$ Playing video games with my friends. (P53)

- $\quad$ Playing video games to take my mind off the COVID-19. (P1803)

- $\quad$ Playing lots of video games with friends. (P83)

Cooking or baking $(n=30)$

- I have been baking a lot. (P1677)

- $\quad$... My mom and I cook together as well. (P880)

- $\quad$ Cooking, healthy eating. (P306)

- I also started learning butterfly knife tricks as a new hobby. (P445)

Arts $(n=72)$

- I've been listening to music more often than not as I find it cheers me up. (P2085)

- I have been doing lots of crafts like making shirts and bracelets. (P220)

- I have been writing and doing things that I like to do that help me feel relaxed and calm. (P803)

- I Iraw a lot, read, listen to music, play a little bit of piano, write a random story. (P249)

Reading ( $n=37)$

- I have been reading ... books have been such a great mood relaxer for me. (P933)

- I have been reading more, journaling. (P1034)

- I also like to read Web comics. (P540)

- $\quad$ Reading stories and books. (P672)

Television $(n=24)$

- Watching Netflix and playing online games. (P94)

- Watching videos of my favourite artist. (P318)

- Mainly distracting myself by watching movies, TV shows. (P1033)

- I have chosen to watch a lot of movies. (P294)

while isolated at home owing to school closures and stay-athome measures. Although the adverse effects of e-learning have not been addressed in this study, ${ }^{26}$ school is an important environment that supports adolescents' ability to cope with 
daily stressors ${ }^{27}$ and can act as a gateway for access to mental health resources. ${ }^{28}$ Participants' objective (e.g., social networks) and subjective support (i.e., emotional experience and satisfaction) diminished when public health interventions were implemented. ${ }^{14,29}$ Low social support is associated with higher incidence of depression and anxiety among adolescents, ${ }^{14,30,31}$ and social support can mediate the association between a stressor and psychological distress. ${ }^{32}$ Therefore, losing this anchor in life not only contributed to adolescents' mental distress but restricted their access to peer support and mental health services at a very critical time. . $^{33,34}$

Though faced with substantial societal disruption, participants recognized positives amid the pandemic, with the most common sentiment around experiencing less stress and pressure from reduced physical, mental and social demands. Some adolescents also identified improved self-reflection and selfcare, as the pandemic allowed time for personal investment. This aligns with research showing that a positive aspect of COVID-19 restrictions was having time to oneself and opportunities to set goals. ${ }^{35}$ Future research is needed assessing the positive associations between the pandemic and adolescents' well-being.

While much research points to the harmful and destructive coping mechanisms that adolescents may engage in during challenging life experiences (e.g., substance use ${ }^{24,36}$ ), our results show that many adolescents were actively adopting positive and healthful coping strategies (e.g., reading, arts and baking). The most prominent strategy mentioned was physical activity. Physical activity has mental health benefits for young people ${ }^{37}$ and is a promising strategy for promoting mental health. ${ }^{38}$ Additionally, many participants used safe peer interactions by connecting with friends and loved ones, both virtually and in person (while maintaining physical distance) as a coping strategy. Maintaining social relations and friendships can be associated with better mental health outcomes among adolescents ${ }^{26}$ and brings attention to social interactions as an important coping strategy for this demographic. ${ }^{34}$ Although adolescents faced a variety of negative emotions and feelings, our results show that some seem to be gravitating toward using positive coping strategies to help them through these uncertain times.

\section{Limitations}

Data were collected during the first wave of the COVID-19 pandemic (June to September 2020); therefore, responses may depict the pandemic situation in the region or province at that time, or if a family member or friend was affected. In addition, although the survey was offered in both English and French, all responses to the open-ended questions were in English, potentially skewing data in favour of 1 language.

This study reports on the responses to 2 open-ended, optional e-survey questions and thus was unable to capture the depth and nuance that more intensive qualitative methods, such as interviews or focus groups, would provide. It is possible that adolescents experiencing more distress would respond to the optional questions with more extreme responses. Given that there was no mention of family-related trauma, participants may have been youth who generally felt safe in their home. Further, study results may be affected by nonresponse bias in that a higher proportion of female than male adolescents completed the survey, and participants required access to a digital device as well as the Internet to be included in the study.

Our findings identify key areas of impact on adolescents' mental well-being, and further qualitative research should be undertaken to explore these in greater detail.

\section{Conclusion}

This youth-informed study identified important ways that the pandemic has affected adolescents' mental and emotional well-being. While results show numerous harms, there is also hope in the form of positive coping mechanisms. Pandemic public health policy should recognize and support young people's positive coping strategies and, most urgently, work to facilitate safe peer interactions.

\section{References}

1. Public Health Agency of Canada COVID-10 Surveillance and Epidemiology Team. A retrospective analysis of the start of the COVID-19 epidemic in Canada, January 15-March 12, 2020. Can Commun Dis Rep 2020;46:236-41.

2. Hawke LD, Monga S, Korczak D, et al. Impacts of the COVID-19 pandemic on youth mental health among youth with physical health challenges. Early Interv Psychiatry 2020 Oct. 20 [Epub ahead of print]. doi: 10.1111/eip.13052.

3. Zhang C, Ye M, Fu Y, et al. The psychological impact of the COVID-19 pandemic on teenagers in China. 7 Adolesc Health 2020;67:747-55.

4. Gruber J, Prinstein MJ, Clark LA, et al. Mental health and clinical psychological science in the time of COVID-19: challenges, opportunities, and a call to action. Am Psychol 2021;76:409-26.

5. Brown BB. Peer groups and peer culture. In: Feldman SS, Elliot GR, editors. At the Threshold: The Developing Adolescent. Cambridge (MA): Harvard University Press; 1990:171-96.

6. Hartup WW. The company they keep: friendships and their development significance. Child Dev 1996;67:1-13.

7. Ryan AM. Peer groups as a context for the socialization of adolescents' motivation, engagement, and achievement in school. Educ Psychol 2000;35:101-11.

8. Prinstein MJ, Giletta M. Peer relations and developmental psychopathology. In: Cicchetti D, editor. Developmental Psychopathology. 3rd ed. New York: Wiley; 2016:527-79.

9. Loades ME, Chatburn E, Higson-Sweeney N, et al. Rapid systematic review: the impact of social isolation and loneliness on the mental health of children and adolescents in the context of COVID-19.7 Am Acad Child Adolesc Psychiatry 2020;59:1218-39.e3.

10. Hawke LD, Barbic SP, Voineskos A, et al. Impacts of COVID-19 on youth mental health, substance use, and well-being: a rapid survey of clinical and community samples. Can 7 Psychiatry 2020;65:701-9.

11. Nathiya D, Singh P, Suman S, et al. Mental health problems and impact on youth minds during the COVID-19 outbreak: cross-sectional (REDCOVID) survey. Social Health and Behavior 2020;3:83-8.

12. Briggs DC, Kattey KA. COVID-19: mental and social health-related complaints among children and adolescents in Nigeria: parents'/caregivers' perception — an online survey. Int Neuropsychiatr Dis 7 2020;14:1-13.

13. Xie X, Xue Q, Zhou Y, et al. Mental health status among children in home confinement during the coronavirus disease 2019 outbreak in Hubei Province, China. 7AMA Pediatr 2020;174:898-900.

14. Qi M, Zhou S-J, Guo Z-C, et al. The effect of social support on mental health in Chinese adolescents during the outbreak of COVID-19. 7 Adolesc Health 2020;67:514-8.

15. Skinner EA, Zimmer-Gembeck MJ. Challenges to the developmental study of coping. New Dir Child Adolesc Dev 2009;(124):5-17.

16. Aldwin CM, Skinner EA, Zimmer-Gembeck MJ, et al. Coping and self-regulation across the life span. In: Fingerman KL, Berg CA, Smith J, et al., editors. Handbook of Life-span Development. New York: Springer Publishing Company; 2011:561-87.

17. Eysenbach G. Improving the quality of Web surveys: the Checklist for Reporting Results of Internet E-Surveys (CHERRIES). 7 Med Internet Res 2004;6:e34.

18. Boak A, Elton-Marshall T, Mann RE, et al. The mental health and well-being of Ontario students, 1991-2019: detailed findings from the Ontario student drug use and health survey. Toronto: Centre for Addiction and Health Health; 2020.

19. Arunkumar K, Bowman DD, Coen SE, et al. Conceptualizing youth participation in children's health research: insight from a youth-driven process for developing a youth advisory council. Children (Basel) 2018;6:3. 
20. Hsieh H-F, Shannon SE. Three approaches to qualitative content analysis. Qual Health Res 2005;15:1277-88.

21. McKenna L, Brooks I, Vanderheide R. Graduate entry nurses' initial perspectives on nursing: content analysis on open-ended survey questions. Nurse Educ Today 2017;49:22-6.

22. Auerbach C, Silverstein LB. Qualitative data: an introduction to coding and analysis. New York: New York University Press; 2003.

23. Hickey G, Kipping C. A multi-stage approach to the coding of data from open-ended questions. Nurse Res 1996;4:81-91.

24. Dixon LJ, Leen-Feldner EW, Ham LS, et al. Alcohol use motives among traumatic event-exposed, treatment-seeking adolescents: associations with posttraumatic stress. Addict Behav 2009;34:1065-8.

25. Zhang $\mathrm{W}$, Liu $\mathrm{H}$, Jiang $\mathrm{X}$, et al. A longitudinal study of posttraumatic stress disorder symptoms and its relationship with coping skill and locus of control in adolescents after an earthquake in China. PLoS One 2014;9:e88263.

26. Marques de Miranda D, da Silva Athanasio B, Sena Oliveira ACS, et al. How is COVID-19 pandemic impacting mental health of children and adolescents? Int 7 Disaster Risk Reduct 2020;51:101845.

27. Lee J. Mental health effects of school closures during COVID-19. Lancet Child Adolesc Health 2020;4:421.

28. Merikangas KR, He J-p, Burstein M, et al. Service utilization for lifetime mental disorders in U.S. adolescents: results of the National Comorbidity Survey-Adolescent Supplement (NCS-A). 7 Am Acad Child Adolesc Psycbiatry 2011;50:32-45

29. Kaniasty K, Norris FH. Longitudinal linkages between perceived social support and posttraumatic stress symptoms: sequential roles of social causation and social selection. 7 Trauma Stress 2008;21:274-81.

30. Guntzviller LM, Williamson LD, Ratcliff CL. Stress, social support, and mental health among young adult Hispanics. Fam Community Healtb 2020;43: $82-91$.

31. Simons HR, Thorpe LE, Jones HE, et al. Perinatal depressive symptom trajectories among adolescent women in New York City. 7 Adolesc Health 2020;67:84-92.

32. Seabrook JA, Avison WR. Socioeconomic status and cumulative disadvantage processes across the life course: implications for health outcomes. Can Rev Sociol 2012;49:50-68

33. Emans SJ, Ford CA, Irwin CE Jr, et al. Early COVID-19 impact on adolescent health and medicine programs in the United States: LEAH program leadership reflections. 7 Adolesc Health 2020;67:11-5.

34. Waselewski EA, Waselewski M, Chang T. Needs and coping behaviors of youth in the U.S. during COVID-19. 7 Adolesc Health 2020;67:649-52.

35. Branquinho C, Kelly C, Arevalo LC, et al. "Hey, we also have something to say": a qualitative study of Portuguese adolescents' and young people's experiences under COVID-19. 7 Community Psychol 2020;48:2740-52.

36. Blumenthal H, Blanchard L, Feldner MT, et al. Traumatic event exposure, posttraumatic stress, and substance use among youth: a critical review of the empirical literature. Curr Psychiatry Rev 2008;4:228-54.
37. Biddle SJH, Asare M. Physical activity and mental health in children and adolescents: a review of reviews. Br 7 Sports Med 2011:45:886-95.

38. Pascoe M, Bailey AP, Craike M, et al. Physical activity and exercise in youth mental health promotion: a scoping review. BMF Open Sport Exerc Med 2020; 6:e000677.

Affiliations: Human Environments Analysis Laboratory (Ferguson, Coen, Tobin, Martin, Seabrook, Gilliland), and Department of Geography and Environment (Ferguson, Tobin, Gilliland), Western University; Children's Health Research Institute (Ferguson, Martin, Seabrook, Gilliland), Lawson Health Research Institute, London, Ont.; School of Geography (Coen), University of Nottingham, Nottingham, UK; Faculty of Health Disciplines (Martin), Athabasca University, Athabasca, Alta.; School of Food and Nutritional Sciences (Seabrook), Brescia University College at Western University, London, Ont.

Contributors: All authors substantially contributed to the conception of the work, interpretation of the data and development of this manuscript. All authors contributed to drafting or revising the manuscript, gave final approval of the version to be published and agreed to be accountable for all aspects of the work.

Funding: Support for postdoctoral and graduate student trainees on this project was provided by the Children's Health Research Institute and the Children's Health Foundation.

Content licence: This is an Open Access article distributed in accordance with the terms of the Creative Commons Attribution (CC BY-NC-ND 4.0) licence, which permits use, distribution and reproduction in any medium, provided that the original publication is properly cited, the use is noncommercial (i.e., research or educational use), and no modifications or adaptations are made. See: https://creativecommons.org/licenses/by-nc-nd/4.0/

Data sharing: Data are not available to others owing to research ethics board requirements.

Acknowledgement: The authors are grateful to members of the Human Environments Analysis Laboratory Youth Advisory Council for inspiring this work and helping to develop and pilot test the survey.

Supplemental information: For reviewer comments and the original submission of this manuscript, please see www.cmajopen.ca/content/9/4/ E1013/suppl/DC1. 